\title{
The Influence of Travelling Fires on a Concrete Frame
}

\author{
Angus Law ${ }^{1}$, Jamie Stern-Gottfried ${ }^{2}$, Martin Gillie ${ }^{3}$, and Guillermo Rein ${ }^{4}$
}

\begin{abstract}
When building fires occur in large, open, compartments they rarely burn uniformly across an entire floor plate of a structure. Instead, they tend to travel, igniting fuel in their path and burning it out as they move to the next fuel package. Current structural fire design methods do not account for these types of fires. This paper applies a novel methodology for defining a family of possible heating regimes to a framed concrete structure using the concept of travelling fires. A finite-element model of a generic concrete structure is used to study the impact of the family of fires; both relative to one another and in comparison to the conventional codified temperature-time curves. It is found that travelling fires have a significant impact on the performance of the structure and that the current design approaches cannot be assumed to be conservative. Further, it is found that a travelling fire of approximately $25 \%$ of the floor plate in size is the most severe in terms of structural response. It is concluded that the new approach is simple to implement, provides more realistic fire scenarios, and is more conservative than current design methods.
\end{abstract}

\section{Introduction}

Since the early $20^{\text {th }}$ century, the Standard Fire test and associated temperature-time curve [1, 2] have been used world-wide to give fire ratings to structural assemblies and to design complete structures [3]. The Standard Fire temperature-time curve was created in an attempt to regulate testing between different laboratories thereby ensuring a uniform standard of safety. However, almost as soon as it was conceived, a number of problems were identified with it. Notably, no account is taken of differences in fuel load, fire compartment size or ventilation conditions, all of which profoundly affect the behaviour of a compartment fire. To address some of these shortcomings, other temperature-time curves have been proposed. Perhaps the most widely known in structural design are the "parametric" fires curves. Initially developed by Pettersson [4], these curves have been modified and are incorporated into the Eurocode structural design standards [5]. They allow design fires to be calculated that, unlike the Standard Fire curve, depend on the fuel load, thermal inertia of linings, and ventilation conditions of a fire compartment. Parametric fires therefore predict more realistic temperature-time curves than the Standard Fire and can be roughly replicated by burning wooden cribs in a small fire compartment. Despite these benefits, parametric fires remain very crude representations of fires in any but the simplest of compartments, as will be described in section 2. Moreover, they are unsuitable for application in the large, open-plan spaces that are a common feature of many modern buildings. Thus, there remain significant shortcomings amongst the traditional design methods for specifying the thermal inputs for use in structural fire design, particularly for large compartments.

By contrast, over the past 20-30 years, knowledge and understanding of how structures respond to elevated temperatures has developed rapidly and to a point where it is now possible to include a large variety of phenomena in structural models and to predict the response of structures subject to known temperature loading with good accuracy [6-8]. Coupled with the recently developed performance-based design codes $[9,10]$, these capabilities have given engineers the freedom to design structures to resist high thermal loadings in innovative, efficient ways.

Thus, while the ability to predict subsequent structural behaviour has reached an advanced level; the thermal inputs used in structural fire design remain simplistic, unchanged, and not representative of actual fire dynamics in large compartments. The various limitations inherent in the traditional design methods mean that it is difficult to justify continuing to develop and use complex structural models when one of the dominating input parameters - thermal loading - remains very crudely defined. Without some development of the method for specifying design fires, it will be impossible to obtain the "consistent level of crudeness" which has been identified as a need within the discipline [11]. In an attempt to rectify the mismatch in the levels of sophistication that are currently used for design fires and the subsequent structural analysis, this paper adopts a new approach [1214]. First, a method of defining design fires that are sufficiently flexible to be applied to any fire compartment is presented and discussed. The method has the key benefits of not assuming a uniform temperature within a large fire compartment and allowing for fires that travel within a compartment. Second, the paper considers the implications of using these new design fires by applying them to the analysis of a concrete framed structure subject to full-floor fires and comparing the predictions of various measures of "structural distress" with those obtained when traditional fire curves are used.

\section{Limitations of Current Design Fires}

Parametric and Standard Fires were validated by test data from small fire compartments that were almost cubic. This test geometry allows for good mixing of the fire gases and so produces a uniform temperature distribution within a compartment.

\footnotetext{
${ }^{1}$ Fire Engineer, Arup, Admiral House, Rose Wharf, 78 East Street, Leeds, UK; email: Angus.Law@arup.com

${ }^{2}$ PhD Student, The BRE Centre for Fire Safety Engineering, The University of Edinburgh, UK; Senior Fire Engineer, Arup, 13

Fitzroy Street, London, UK; email: Jamie.Stern-Gottfried@arup.com

${ }^{3}$ Lecturer, The BRE Centre for Fire Safety Engineering, The University of Edinburgh, UK; email: M.Gillie@ed.ac.uk

${ }^{4}$ Lecturer, The BRE Centre for Fire Safety Engineering, The University of Edinburgh, UK; email: G.Rein@ed.ac.uk, reingu@gmail.com
} 
These conditions do not exist in real fires [15] and consequently limitations must be placed on the form of compartment in which the traditional fire curves may be used. For example, Eurocode 1 states that the parametric curves are only valid for compartments with floor areas up to $500 \mathrm{~m}^{2}$ and heights up to $4 \mathrm{~m}$, the enclosure must also have no openings through the ceiling, and the compartment linings are restricted to having a thermal inertia between 1000 and $2200 \mathrm{~J} / \mathrm{m}^{2} \mathrm{~s}^{1 / 2} \mathrm{~K}$, which means that highly conductive linings such as glass facades and highly insulating materials cannot be taken into account. As a result, common features in modern construction like large enclosures, high ceilings, atria, large open spaces, multiple floors connected by voids, and glass façades are excluded from the range of applicability of the current methodologies.

A recent survey of buildings in Edinburgh, UK [16] underlines the implications of these limitations on the applicability of design fires, particularly for modern structures. For buildings built over a long period of time starting in the early 20 th century, $66 \%$ of their total volume falls within the limitations. However, in a newly constructed, modern building that has open spaces and glass facades, only $8 \%$ of the total volume is within the limitations. This suggests that modern building design is increasingly producing buildings that contain compartments to which parametric fires should not be applied.

Additionally, an assumption that has remained unquestioned with each temperature-time curve no matter how they have been applied has been that of uniform burning and uniform compartment temperature. It is assumed that every part of a structural element or compartment is uniformly subject to the same temperature - as defined by the temperature-time curve adopted. Although it may be possible to replicate these conditions in a furnace, a recent experimental review of post-flashover tests [15] has clearly demonstrated that temperature conditions are non-uniform in most compartments. Moreover, the major fires at the Windsor Tower [17], World Trade Center [18, 19] and TU Delft [20] have shown that fires tend to travel around large compartments rather than burn uniformly. Tests have also shown the there is a high degree of temperature variation even within small compartments [21-23].

Therefore, at present, designers are forced to either use parametric fires in compartments for which they are not strictly applicable, apply very onerous and unrealistic Standard Fires to large compartments, or to resort to CFD models of fires in large compartments that are labour intensive to produce. There is a clear need, then, to address the limitations of the currently available design fires if modern performance-based design is not to be restricted.

\section{Travelling Fires}

In light of the various limitations outlined above, a new method for estimating compartment fire temperatures based on the fundamental fire dynamics of the compartment has been proposed [12, 23, 24]. This new method will be used throughout this paper. It uses two temperature fields to represent the gas temperature in a compartment: a high temperature in the flaming region of the fire (the near field); and a cooler temperature for the rest of the compartment (the far field). This approach provides a flexible technique whereby a large range of possible fires in any compartment can be represented. For example, a fire which engulfs an entire large floor plate simultaneously, as in traditional design methods, can be represented, as well as a small fire that travels slowly from one end of a compartment to the other. The full range can then be explored by parametrically varying the size of the fire. This avoids the weakness of previous methods assuming that arbitrary events lead to particular fire conditions, such as assuming that glazing failure leads to one single temperature-time definition for an entire region. Instead, consideration of a wide range of possible fire sizes covers for the inherent variable nature of real fire events (outcome of the combination of particular ignition location, fuel distribution and ventilation conditions). Thus, a family of fires is created ranging from a small travelling fire that burns for a long duration as it travels, to a fire uniformly burning over the full extent of the floor for a shorter time period. Therefore, the method addresses the two key shortcomings of existing methods - restrictions on the nature of applicable fire compartments and the assumption of uniform gas temperatures within a compartment - while still being sufficiently concise for use in structural design.

\subsection{Temperature Definition}

The new design approach represents the horizontal temperature distribution of a fire compartment by means of "near field" and "far field" regions (Figure 1). The near field is the flaming region of the fire. Peak values in small fire have been measured in the range from 800 to $1000^{\circ} \mathrm{C}$ [25] but temperatures of $1200^{\circ} \mathrm{C}$ have been measured for larger enclosure fires [5]. This maximum value of $1200^{\circ} \mathrm{C}$ is chosen for the near field to represent the worst case conditions. The far field represents the temperature of the hot gases away from the flaming region. Far field temperatures can be calculated using any engineering tool that gives temperature distributions away from the fire, including hand calculations or computer modelling. For this study, the simple ceiling jet correlation developed by Alpert has been used [26].

$$
T_{\max }-T_{\infty}=\frac{5.38(\dot{Q} / r)^{2 / 3}}{H}
$$

where $T_{\max }$ is the maximum temperature within the ceiling jet $(\mathrm{K}) ; T_{\infty}$ is the ambient temperature $(\mathrm{K}) ; \dot{Q}$ is the heat release rate $(\mathrm{kW}) ; r$ is the distance from the centre of the fire $(\mathrm{m})$; and $H$ is the floor to ceiling height $(\mathrm{m})$. This correlation was developed for a stationary fire during steady-state conditions but is valid for travelling fires because the flame spread rate $(\sim 0.01 \mathrm{~m} / \mathrm{s}[5])$ is 
much lower than the velocity of the smoke $(\sim 1 \mathrm{~m} / \mathrm{s})$. Thus, the far-field temperature distribution in Eq. (1) moves with the fire in a quasi-steady state form.

As the fire consumes the available fuel and ignites new material in its path, it moves around the floor-plate. Consequently, the gas temperature adjacent to any given structural element is constantly changing as the fire travels both near that element and remote from it. To make the amount of information passed to a structural analysis managable, the monotonically decreasing far field temperature distribution from Alpert's correlation is reduced to a single characteristic value, $T_{f f}$. To do this, the far field temperature is taken as the fourth-power average of $T_{\max }$ (to favour high temperatures in a bias towards radiation heat transfer and onerous structural conditions) over the distance between the end of the near field, $r_{n f}$, and the end of the far field, $r_{f f}$. This average is calculated by Eq. (2).

$$
T_{f f}=\frac{\left[\int_{r_{n f}}^{r_{f f}}\left(T_{\max }\right)^{4} d r\right]^{1 / 4}}{\left(r_{f f}-r_{n f}\right)^{1 / 4}}
$$

Figure 1 demonstrates the concept of a near field and a far field for a travelling fire. Any given location is exposed to the far field temperature for a period before the arrival of the flaming, near field region. After all the fuel at the location has been consumed and the near field moves away, it is then subjected to the far field temperature again until all the fuel in the entire compartment has been consumed, at which point the temperature returns to ambient and the structure cools.
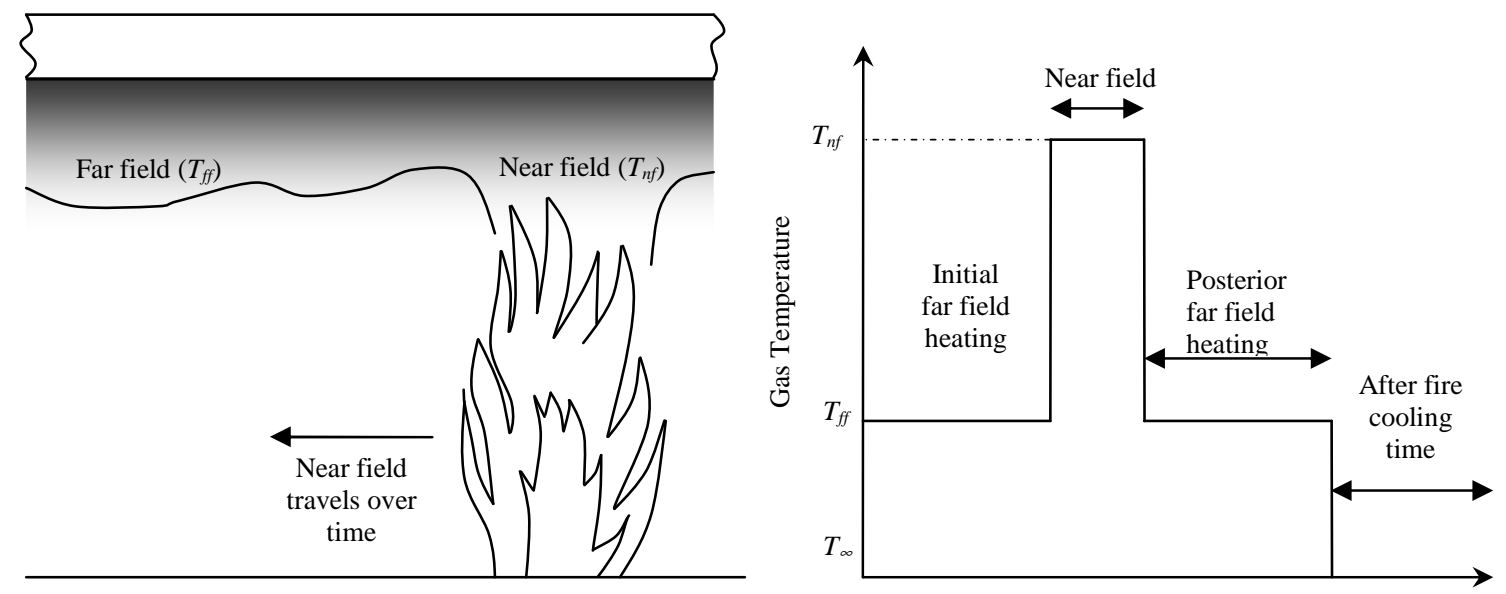

Figure 1. (a) Near field and far field temperatures induced by localized travelling fire; (b) far field and near field exposure durations at an arbitrary point within the fire compartment.

\subsection{Fire Size}

The flexibility of the method stems from parametrically varying size, shape, and path followed by the fire. It is assumed that, once alight, any area of the floor plate will continue to burn at the same rate until all the fuel is consumed. The local burning time for any fire size can, therefore, be simply calculated from the fuel load and the heat release rate. Once the local fuel is burnt out, the fire will move to a new area. After the fire has travelled around the whole compartment, the cooling of the structure takes place. The fire size is varied, in this study from $1 \%$ to $100 \%$ of the compartment floor area. Assumptions and details of how to calculate the resultant heating from this method can be found in other papers by the authors $[13,14,23]$.

\section{Structural Failure Criteria}

The methodology presented above can be used to study the impact of different travelling fires on the response of a structure. However, without a means to compare the structural response, it is impossible to draw any conclusions. There are many different methods of assessment available for fire-affected structures of varying degrees of complexity.

The simplest and most widely used measure of structural distress is maximum deflection. Typically, failure is defined as a ratio of deflection (e.g. span/20 [2]). The allowable deflection does not represent a value at which an assembly catastrophically loses stability; rather, it is the maximum deflection allowable in a furnace test in order to protect expensive experimental equipment. In spite of this, deflection is a simple and useful measure which can be used to give some indication of structural distress. It is possible to use the relative deflections caused by different fires as a means for comparison.

Another simple measure of performance for concrete structures is the maximum temperature of the tension reinforcement. Failure in steel members is often said to have occurred when the axial capacity of a section is half its ambient capacity. For reinforcing steel in concrete, this critical temperature is typically taken as $593^{\circ} \mathrm{C}[27]$. Again, although this is a fairly arbitrary measure of 
"failure", the temperature of the rebar offers a simple and easily comparable metric that can be used to compare the impact of different fires on a structure.

The ultimate strain in the tension reinforcement is also often used as a definition of failure; beyond this strain, the rebar can be assumed to have failed. This measure is better suited to the numerical analysis of structures rather than fire tests because of the difficulties associated with instrumentation of rebar. However, the strain in the tension steel provides another measure which can be used to compare the relative impact of the different fires. The ultimate strain for steel at any temperature is typically taken as $0.2[10,28]$.

\section{Structural Modelling}

The remainder of this paper is a case study that demonstrates how the above travelling fire methodology and failure measures can be applied in a structural analysis. Initially, a number of "base case" scenarios are considered and the differences between the predicted structural responses compared; a parametric study is then conducted to assess the validity and effect of the various assumptions made by the new approach. Finally, the impact of the shape and path of the fire is considered.

\section{1}

\section{Structural Arrangement}

The case study analyses the impact of travelling fires on a generic concrete office building. The structure is a nine storey, flat-slab concrete frame, designed in accordance with the Eurocodes [29-31]. A plan and elevation of the structure are shown in Figure 2. The floor slabs are 200mm thick; the interior columns 400x400mm; and the exterior columns 300x300mm. The design strength of the concrete in the columns is $48 \mathrm{MPa}$, and that in the slabs $40 \mathrm{MPa}$. In this paper fires burning on the fourth floor are considered. This allows the structural effects of a mid-level fire to be analysed without the need to explicitly consider effects of the foundations or the building's top storey.
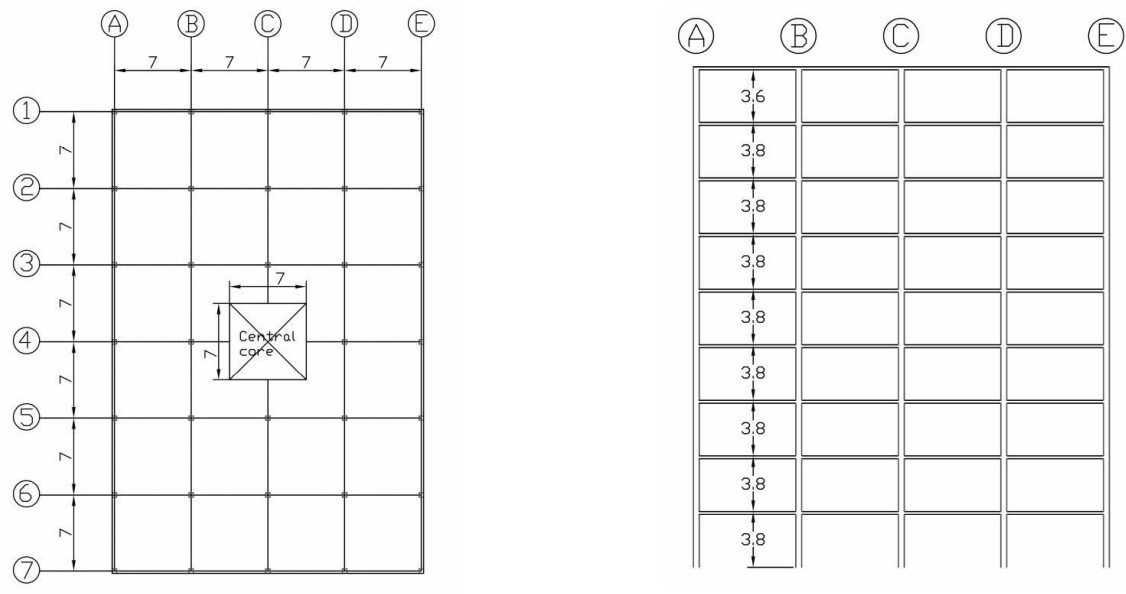

Figure 2. Plan and elevation of concrete structure, dimensions in metres.

Two finite-element models of the central floors of the structure were created using the commercially available Abaqus [32] software. One model was a heat-transfer model developed to determine structural temperatures, the other a stress analysis model produced to predict the mechanical response of the structure. The models were sequentially coupled so the heat-transfer analysis results affected the mechanical response. Both models extended from the base of the columns at the third-storey level, to the top of the columns at the fifth-storey level. The floor slabs were modelled using shell elements, the columns using three-dimensional solid elements and the rebar using truss elements.

In the heat-transfer model, thermal properties were specified in accordance with those of a $1.5 \%$ moisture content concrete, as defined in Eurocode 2 [9]. Heating of the structure was analysed by applying relevant radiation and convection boundary conditions to the surface of the structure. For the purposes of this study, an emissivity of 0.7 and a convective coefficient of $25 \mathrm{~W} / \mathrm{m}^{2} \mathrm{~K}$ were assumed in accordance with Eurocode guidance [9].

For the mechanical analysis, all of the material properties used in the model were temperature dependent and in accordance with Eurocode 2, and the yield criterion used for the concrete was the "damaged plasticity" model, based on the work of Lubliner [33]. A series of mesh sensitivity studies were conducted to find the optimum mesh density. The final mesh density used was $8 \times 8 \times 18$ elements per floor per column, and an average element size of $0.4735 \mathrm{~m}$ in the slab.

The base of each column was assumed to be fixed in translation and rotation, and the top of each column was fixed in all directions other than vertical. As the higher storeys of the structure were not modelled, the equivalent loads that would have been transferred into the column heads were calculated using a full-frame elastic model and applied to the remaining structure during the loading phase of the analysis. The central core of the building was not modelled explicitly but was assumed to provide rigid support to the adjoining structure. 


\section{Base Case Fires}

The "base case" family of fires were defined as fires that travelled linearly from one side of the structure to the other (Figures 3 and 4). The fire sizes considered were: $1 \%, 2.5 \%, 5 \%, 10 \%, 25 \%, 50 \%$ and $100 \%$ of the floor area. It was assumed that the fuel load $\left(q_{f}\right)$ was $570 \mathrm{MJ} / \mathrm{m}^{2}$; the heat release rate per unit area $\left(\dot{Q}^{\prime \prime}\right)$ was $500 \mathrm{~kW} / \mathrm{m}^{2}$.The distance to the far field for Alpert's equation was measured from the centre of the fire at the mid-point of the building along the direction of fire travel as shown in Figure 5. This creates the shortest far field distance, which in turns leads to the highest far field temperature, possible for that specific scenario. This is done to err on the side of conservatism. Figure 5 shows the distances to the end of the near field and to the end of the far field for both the case where the near field is smaller than the core and the case where it is larger. The near field distance is simply calculated from the geometry of the structure and the fire area for each case.

The fuel conditions above resulted in a local burning time of $19 \mathrm{~min}$ for any single area. For example, as there were four phases in the $25 \%$ fire size, it lasted for a total burning duration of $76 \mathrm{~min}$, and had a far field temperature of $805^{\circ} \mathrm{C}$. The near field temperature is taken as the flame temperature, as assumed to be $1200^{\circ} \mathrm{C}$ [13]. The $2.5 \%$ fire size, meanwhile, had a total burning duration of $760 \mathrm{~min}$ and a far field temperature of $325^{\circ} \mathrm{C}$. Figure 3 shows the total burning duration and far field temperatures for each of the base case fires. It should be noted for the $100 \%$ fire size, the far field temperature is the same as the near field temperature.

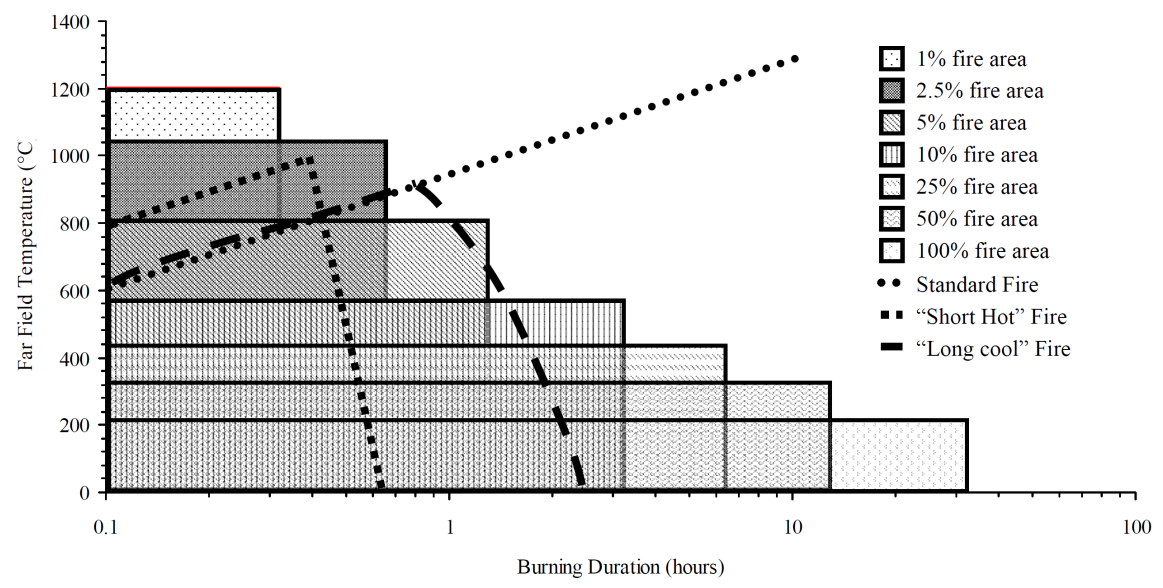

Figure 3. Far field temperatures vs. total burning durations for different fire sizes. Standard and two ("short hot" and "long cool") parametric Eurocode fire curves are also shown for reference.
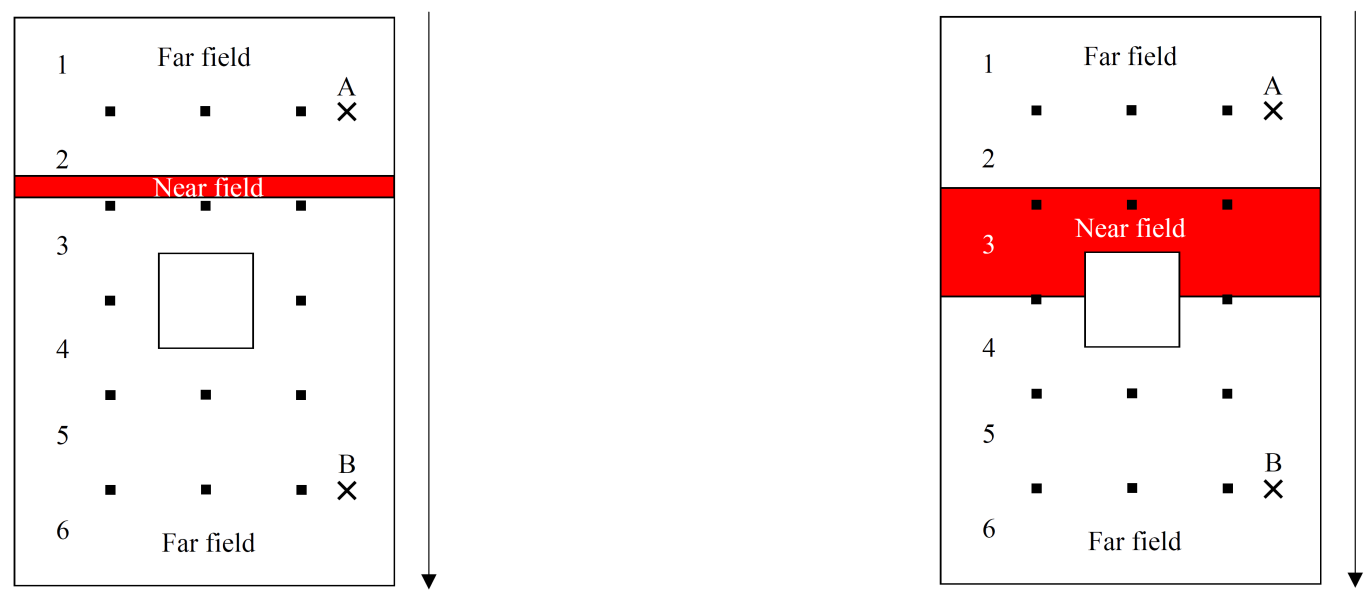

Figure 4. (a) Progression of the $2.5 \%$ fire across the floor plate; (b) Progression of the $25 \%$ fire across the floor plate. Bay numbers are indicated in both figures.

Thermal and structural analyses were conducted using the finite-element model described above. To allow meaningful conclusions to be drawn from the modelling, it should be noted that the analyses were intended to be comparative. Therefore, for the remainder of this paper, the metrics that will be used to quantify the response of the structure will be the three simple measures discussed above - temperature, strain in the tension steel, and central deflection of each bay. 
Figure 4 shows the location of the near field part of the way through the $2.5 \%$ and $25 \%$ fire sizes. The heat transfer analyses allowed the temperature in the slab soffit rebar to be monitored. Figure 6 shows the gas temperatures and corresponding rebar temperatures for points A and B (indicated in Figure 4) during the 10\% fire. The influence of the near field on the rebar can be clearly seen as a temporary increase in temperature. The prolonged exposure of point B to the far field prior to the arrival of the near field causes the overall peak temperature to be higher than that at point A. Figure 7a shows a similar plot of the temperature profiles for the soffit rebar at the centre of bays 1-6 for the 5\% fire size. It can be clearly seen that the final bay to be subjected to the near field experienced the highest temperature; the long pre-heat induced a higher maximum temperature in this bay, which caused it to be most critical by this metric. This trend was the same with each of the base case fires.

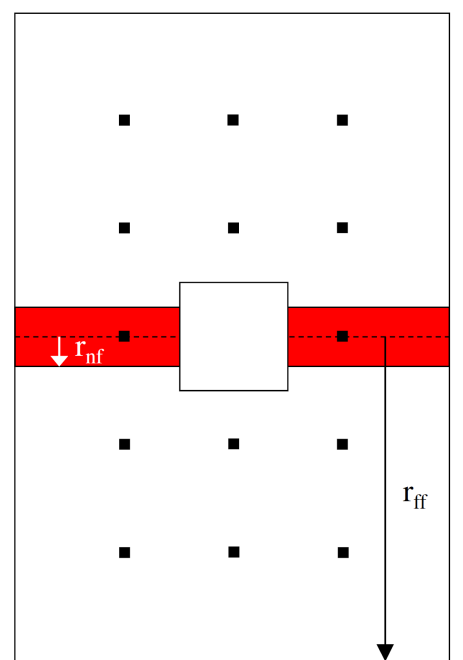

a)

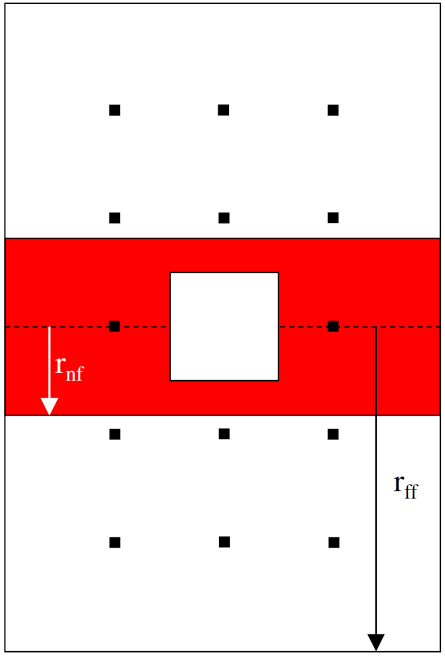

b)

Figure 5. The measurement of $r_{\mathrm{ff}}$ and $\mathrm{r}_{\mathrm{nf}}$ for two different indicative fire sizes (a) small; and (b) large.
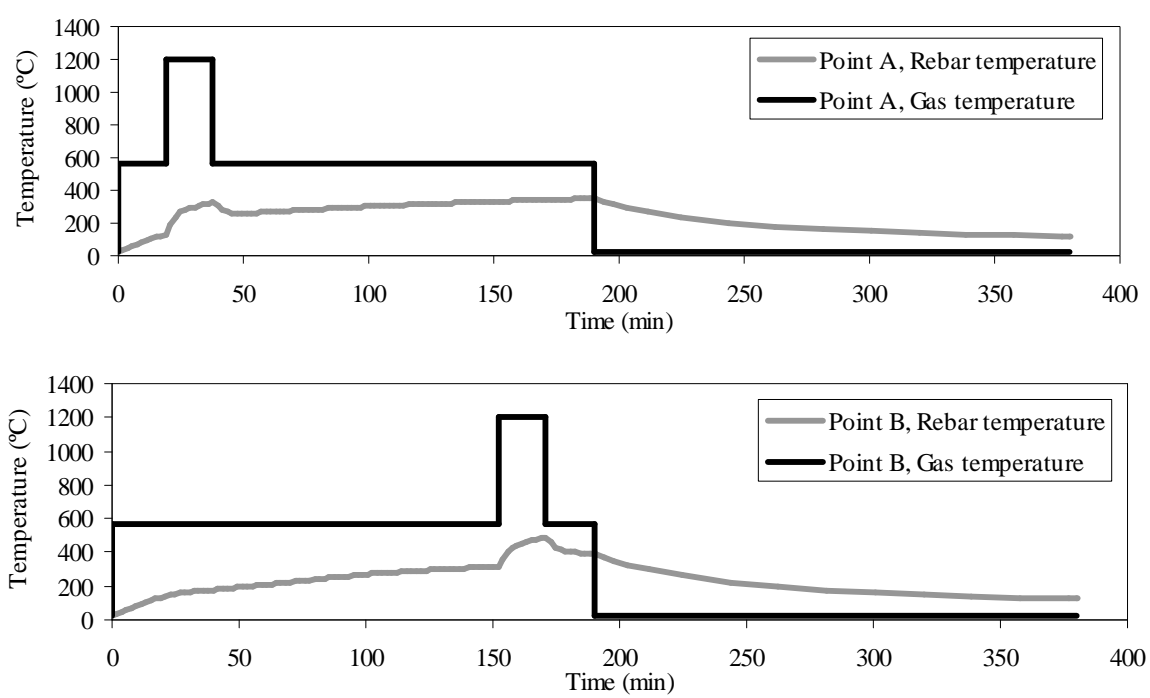

Fig.ure 6. (a) Gas temperature and corresponding rebar temperature at point A; (b) gas temperature and corresponding rebar temperature at point B for a $10 \%$ linearly traveling fire.

Figure $7 \mathrm{~b}$ shows the average temperature in the soffit rebar for each bay. Because the near field of the $5 \%$ fire size does not cover the whole area of any bay simultaneously, the average rebar temperatures are lower. The bay average lower rebar temperatures are a more representative measure of structural vulnerability as they will not be distorted by localized heating effects. For example, were a localized fire to heat only a tiny area of the bay, it would have minimal impact on the overall structural behaviour, but would induce high rebar temperatures. Thus, the bay average lower rebar temperatures will be used as the measure of rebar temperature for the remainder of this paper rather than point temperatures. 
A Law, M Gillie, J Stern-Gottfried, G Rein, The Influence of Travelling Fires on a Concrete Frame, Engineering Structures 33, pp. 1635-1642, 2011. http://dx.doi.org/10.1016/j.engstruct.2011.01.034
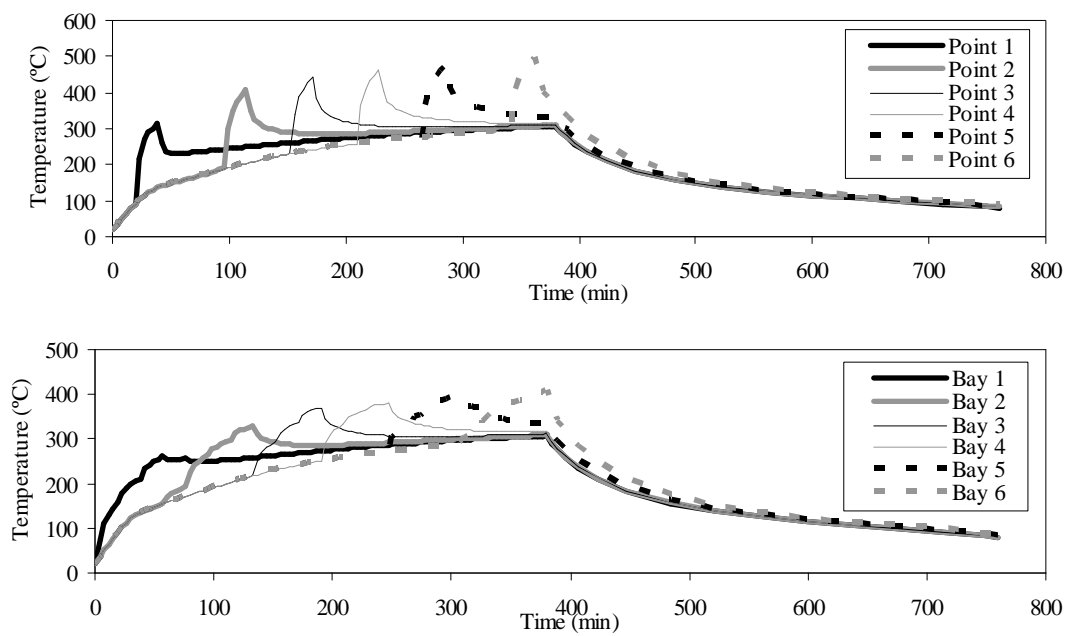

Figure 7. (a) Single point rebar temperature at the centre of bays 1-6 during the 5\% base case fire; (b) average rebar temperatures for the whole of bays $1-6$ for the $5 \%$ base case fire.

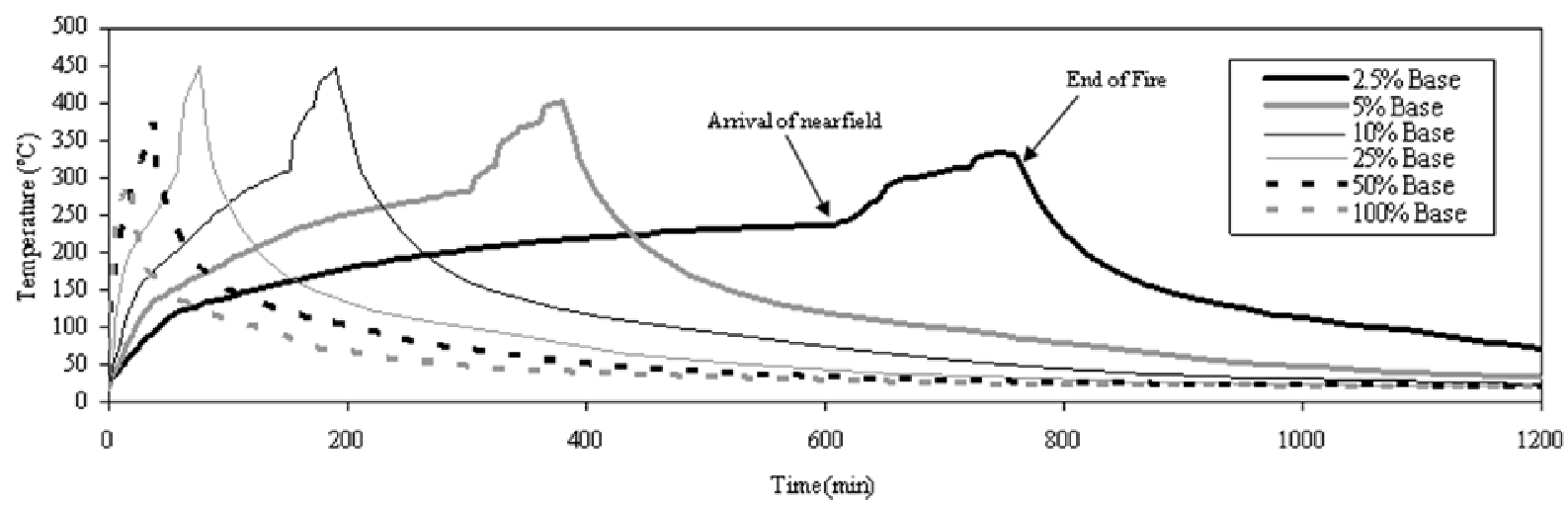

Figure 8. Temperature profiles for the average rebar in the final bay to be heated during the base case fires.
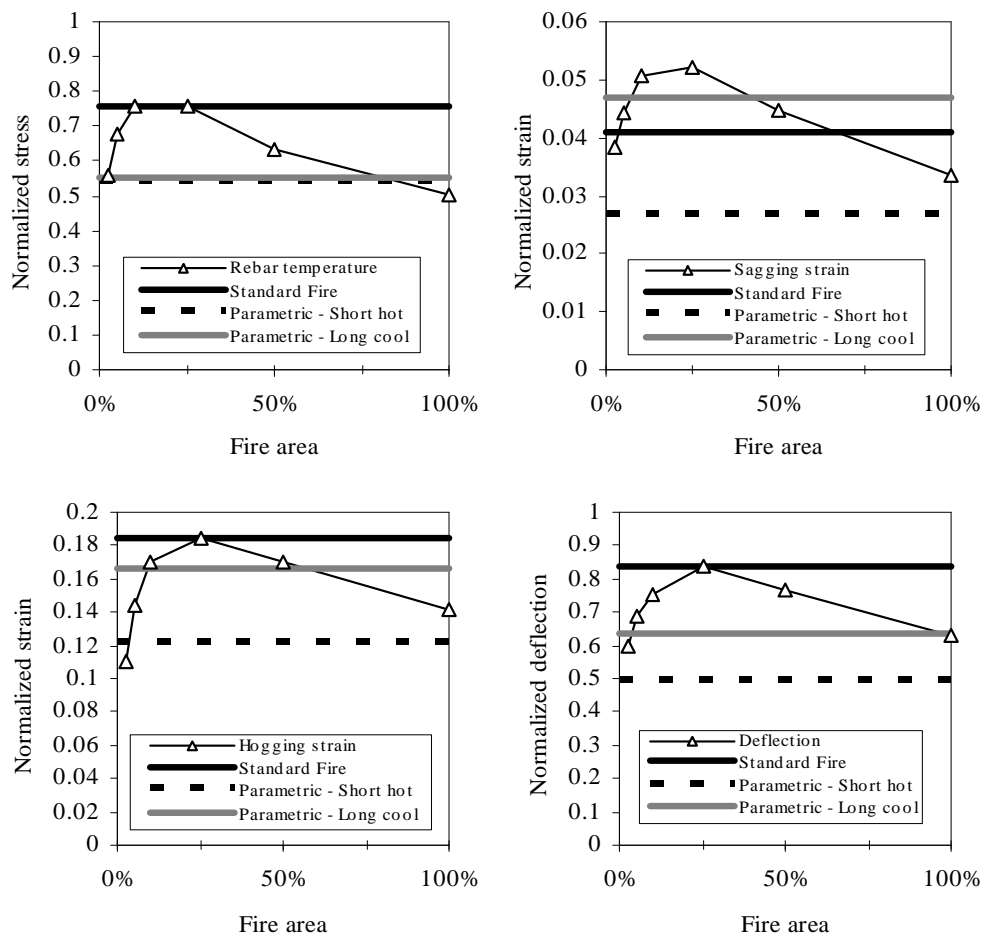

Figure 9. Change in structural distress with near field area: (top left) rebar temperature, Standard Fire equivalent is $1 \mathrm{~h} 37$ min; (top right) sagging tensile strain, value for Standard Fire given after 3 h; (bottom left) hogging tensile strain, Standard Fire equivalent is $1 \mathrm{~h} 18 \mathrm{~min}$; and (bottom right) deflection, Standard Fire equivalent is $1 \mathrm{~h} 54 \mathrm{~min}$. 
A comparison of the rebar temperatures induced in the final bay by the different fires in the family (Figure 8) shows clearly that the highest temperatures are caused by the medium duration fires: $10 \%$ and $25 \%$ fire sizes. For the $2.5 \%$ fire the arrival of the near field at bay 6 is labeled, as is the end of the fire.

A similar process was conducted for each of the structural measures. The absolute value of each measurement technique can be normalized with respect to the appropriate failure definition: $593^{\circ} \mathrm{C}$ for rebar temperature, span/20 for deflection, and 0.2 for rebar strain. It is possible therefore to observe how the level of structural distress varies with each curve in the family of fires. Figure 9 shows the trends for each of the measures against fire size. As a comparison the structure was also subjected to a Standard Fire, a "short hot" parametric fire and a "long cool" parametric fire. The "short hot" fire had a peak temperature of $989^{\circ} \mathrm{C}$ and a total fire time of $37 \mathrm{~min}$, and the "long cool" fire had a peak temperature of $915^{\circ} \mathrm{C}$ and a total fire time of 145 min. Both curves were generated by the parametric temperature-time from Eurocode 1 [31] for the building being examined, varying the assumed glass breakage in the façade for the ventilation factor. The short hot fire assumed $100 \%$ glazing failure along the façade while the long cool fire assumed $25 \%$.

The $25 \%$ fire size induced the highest degree of structural distress in each of the failure metrics. The trend in every metric was the same: the medium sized fires $(5 \%, 10 \%$ and $25 \%)$ caused a higher degree of structural distress than both the smaller and the larger fire sizes fires. It is also notable, that the temperature and deflection measures show the structure as much closer to "failure" than the strain measures. For each measure, a comparison with Standard and parametric fires is also made. The parametric fires universally induced less extreme structural conditions than the medium fire size "base case" scenario. The worst case travelling fire was equivalent to $1 \mathrm{hr} 37 \mathrm{~min}$ of the of a Standard Fire in terms of rebar temperature, $1 \mathrm{hr} 18 \mathrm{~min}$ for hogging tensile stain and $1 \mathrm{hr} 54 \mathrm{~min}$ for deflection. In contrast, the sagging strain was less than that obtained during most of the base case and "long cool" fires; this was because there was no cooling phase during the standard fire so the structure was not pulled into tension.

The results of the base case fires, and their comparison with the codified fires, have shown that the traditional design methods do not necessarily produce the most onerous case for the structure. Indeed a travelling fire based on basic fire dynamics can induce a worse structural scenario. This is in agreement with previous work for steel structures [12, 34]. It has been shown that the medium size (and duration) fires induce the most extreme structural response; the very short fires with a large fire size and the very long fires with a small fire size are less severe for the structure. Specifically the $25 \%$ area fire produced the worst case for the structure. It has also been found that the lack of a cooling phase in the Standard Fire does not allow all the forces that are likely to develop over the course of a real fire to develop; it cannot, therefore be considered conservative [35].

\section{Parametric Study}

A parametric study was conducted to establish the effect of the various assumptions made in the travelling fire methodology on the predicted structural response. As the $25 \%$ fire was found to be the most severe by every metric for this structure, this fire size was used throughout the parametric study.

\subsection{Variation of Far Field Definition}

First, the method used to define the far field temperature was varied, and the response of the structure was monitored using the same metrics that were used in the previous section. The cases studied are described below and illustrated in Figure 10.

1. Single far field (base case). As with the previous analyses, Alpert's far field temperature profile was reduced to a single value by fourth power averaging. The progress of the fire was assumed to move suddenly, i.e. it would jump from one quarter of the floor plate to the next after each burning time. This assumption means the fire is in four specific locations (for the $25 \%$ area fire) over the total burning duration.

2. Two far fields. Rather than reducing the far field to a single value for both sides of the burning area, two separate far fields were assumed, one on either side of the fire. Each far field had a unique temperature defined with the fourth power average.

3. Alpert's temperature profile (sudden). Rather than averaging Alpert's temperature profile as above, the continuous temperature profile defined by Alpert's equation (Eq. 1) was directly applied to the structure. As with the base case fire, the fire moved suddenly from area to area as the fuel was consumed.

4. Alpert's temperature profile (gradual). Alpert's temperature profile was used to define the far field, but the fire was assumed to progress gradually across the structure, rather than jumping suddenly from one area to the next.

The results in Figure 11 show that there is little variation in the performance metrics between the different approaches of defining the far field temperature. Of the different proposed profiles, the "Alpert - sudden" induced the greatest distress in terms of deflection and hogging tension strain. However, the values were only $0.5 \%$ and $3.6 \%$ in excess of the "base case" value respectively. In terms of temperature, the "base case" gave the greatest deflection by a marginal amount $(0.1 \%)$ and the total variation between the largest and smallest temperature was $10.6 \%$. The largest value in terms of the hogging tensile strain was obtained during the "Alpert - gradual" case. For this profile, the maximum strain measured was $4.7 \%$ larger than the "base case" equivalent. 


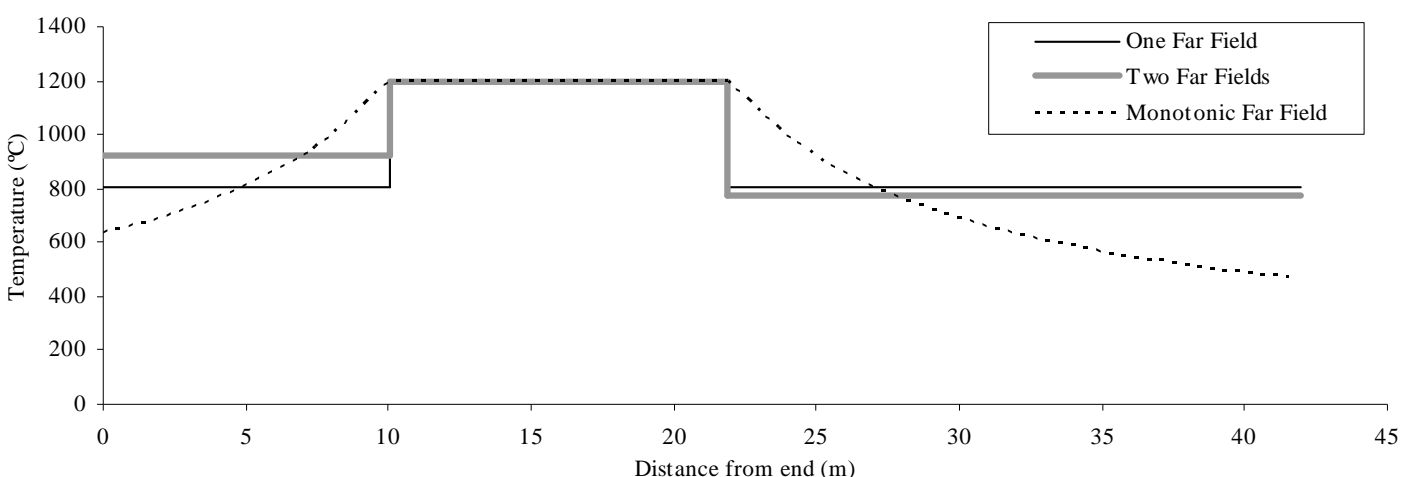

Figure 10. Example range of far field temperature definitions.

This study shows that the variations induced by the different fires in the most critical structural measures are negligible. The variation in the less distressed measures was slightly larger, but still remained small $(<5 \%)$. It therefore appears reasonable that the use of the simple, averaged, temperature profile, i.e. the base case, for the whole of the far field temperature region provides appropriate results and a higher level of detail is not needed. This makes the temperature definitions in the heat transfer model significantly simpler to apply: a key consideration for the use of such an approach in a design context.

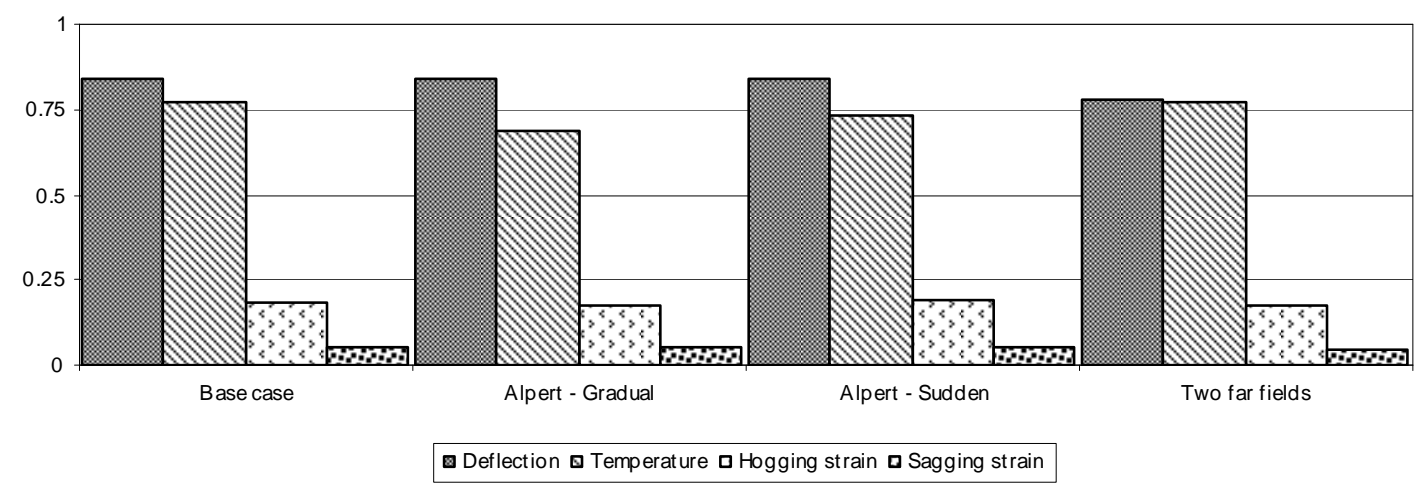

Figure 11. Effect of far field definition on each metric.

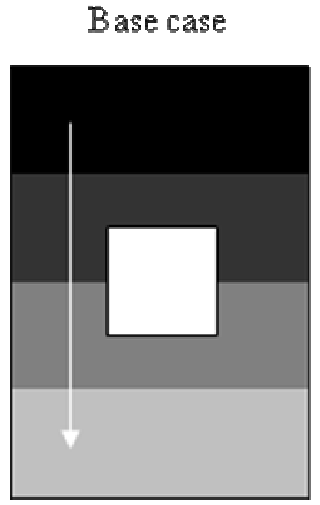

$1^{\text {st burn region }}$
Somer

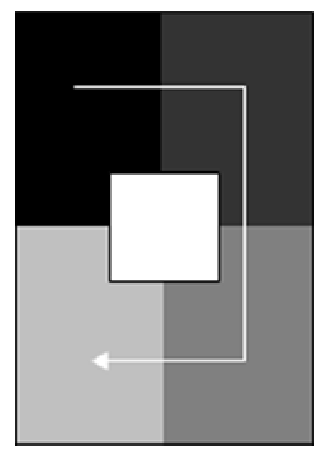

$2^{\text {nd }}$ bum region
Ring - inwards

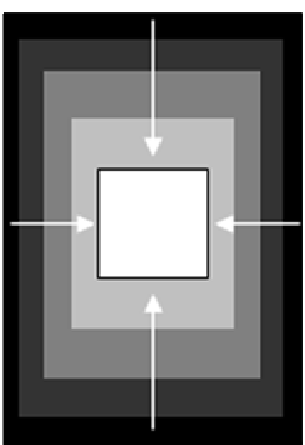

$3^{\text {xd }}$ burn region
Ring - outwards

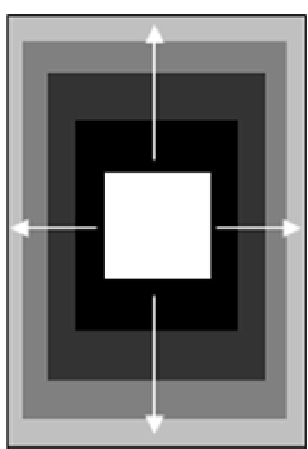

$4^{\text {th }}$ burn region

Figure 12. Different fire shapes and paths. 


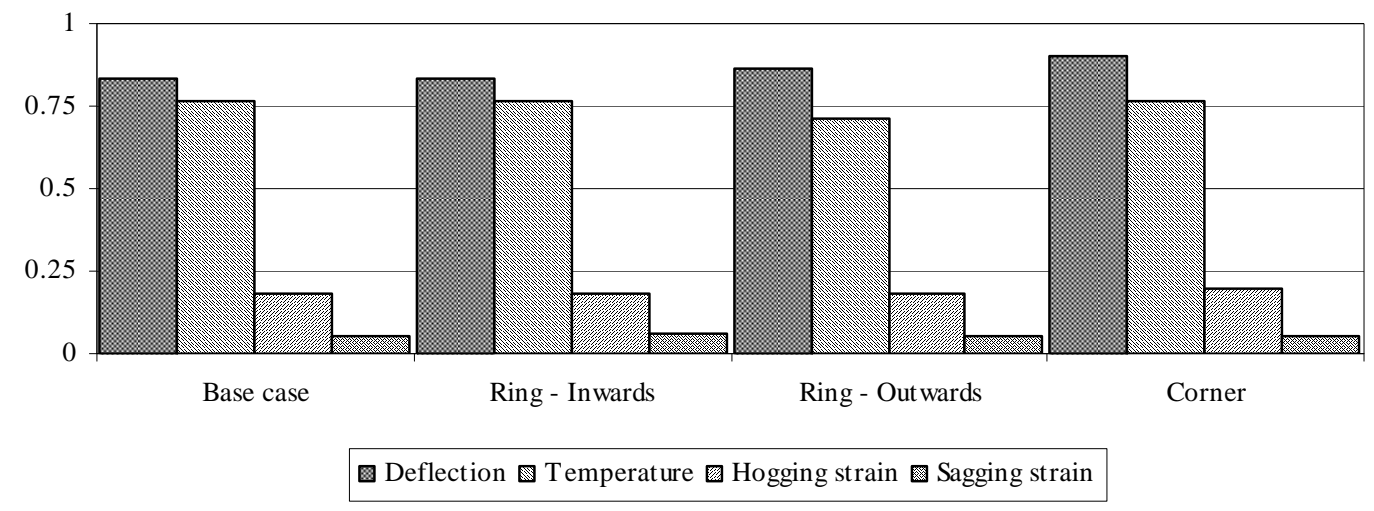

Fig. 13. Influence of path on failure metrics.

\subsection{Fire Path and Shape}

The base case fire described above started at one end of the structure and then progressed linearly across the floor-plate. A real fire could follow a number of possible paths and it has long been recognised that to examine every possible fire scenario would be unfeasible due to the large number of analyses required [3]. However, since the advent of modelling techniques such as the finiteelement method it has become possible to evaluate a number of different structural scenarios quickly. This paper has developed a number of fires and applied them to the same structure. In an attempt to quantify the impact that different fire paths and shapes have on the structure, this study analyses the effect of three other possible fire patterns with a fire size of $25 \%$ of the floor area. In addition to the linear base case, the different fire shapes are illustrated in Figure 12 and are described below:

Corner fire. Initiated in one corner of the structure and spread around the building's core. Due to symmetry, results are the same for clockwise and anti-clockwise fires.

Ring fire, Outwards. Initiated as a ring around the core, and spread concentrically outwards.

Ring fire, Inwards. Initiated in a peripheral ring around the edge of the structure, and spread concentrically inwards towards the core.

The results were broadly similar with some metrics showing an increase and some showing a decrease but there is some variation between the different fires paths. The corner fire was found to be the most severe scenario. The relative increase in comparison to the base case model was $8 \%$ for deflection; $5 \%$ and $10 \%$ for hogging and sagging strain respectively; and $0 \%$ for the rebar temperature. Figure 13 shows the difference between the four fire shapes analysed. Therefore it can be concluded that the shape and path of the fire does have a small impact on the response of the structure.

\section{Summary and Concluding Remarks}

A comparative analysis of the impact of a number of different design fires on a concrete frame has been conducted. A new approach to defining temperature-time curves for design has been presented. The relative impact of the conventional codified curves and the new "travelling fire" methodology has been studied.

The "travelling fire" approach is based on observations from real, large building fires, and founded on the fundamental fire dynamics of a large open plan floor plate. It allows a range of realistic fires to be considered and, thus, allows structural engineers to better understand how different fires might affect the behaviour of a building. Though based on complex temperature distribution data, a simplified approach allows a single value far field temperature distribution. It has been demonstrated that this simplification is a good approximation to more complex temperature fields obtained from fundamental fire dynamics. The simplified far field approach is easily implemented in finite-element codes.

The generic concrete frame which was subjected to the various fires was the same in each of the analyses. It has thus been possible to draw strong comparative conclusions, particularly given the variety of measures used to assess the structure, which include

- Travelling fires have a more severe impact on the performance of this structure than the Eurocode parametric fires. The Eurocode fires cannot, therefore, be considered conservative.

- The fires of medium duration and fire size are the most severe in terms of their impact on the structure.

- The $25 \%$ fire size fire was conclusively found to be the most severe by every measure used.

- The assumption of a simplified far field temperature was valid: more complex and realistic temperature profiles had little impact on the overall structural behaviour. 
The authors would like to gratefully acknowledge BRE trust, Arup, and the EPSRC for supporting and funding this work.

\section{References}

[1] ASTM E 119 - 00a Standard Test Methods for Fire Tests of Buildings Construction and Materials, 2000.

[2] BS476-20:1987. Fire Tests on Buildings Materials and Structures - Part 20: Method for Determination of the Fire Resistance of Elements of Construction: BSI, 1987.

[3] Babrauskas V, Williamson RB. The historical basis of fire resistance testing — Part II. Fire Technology 1978;14:304.

[4] Pettersson O, Magnuson SE, Thor J. Fire Engineering Design of Structures. Publication 50: Swedish Institute of Steel

Construction, 1976.

[5] Drysdale D. An Introduction to Fire Dynamics. Chichester: Wiley, 1998.

[6] Franssen JM, Cooke GME, Latham DJ. Numerical simulation of a full scale fire test on a loaded steel framework.

Journal of Constructional Steel Research 1995;35:377.

[7] Bailey CG, Burgess IW, Plank RJ. Computer Simulation of a Full-Scale Structural Fire Test. The Structural Engineer 1995;74:93.

[8] Gillie M, Usmani AS, Rotter JM. A Sturctural Analysis of the Cardington British Steel Corner Test. Journal of Construction Steel Research 2002;58:427.

[9] EN1992-1-2. Eurocode 2: Design of Concrete Structures - Part 1-2: General rules - Structural fire design, 2004.

[10] EN1993-1-2. Eurocode 3: Design of Steel Structures - Part 1-2: General rules - Structural fire design, 2005.

[11] Buchanan A. The Challenges of Predicting Structural Performance in Fires. Ninth International Symposium for Fire Safey Science, vol. 9. Karlsruhe, 2008.

[12] Stern-Gottfried J, Rein G, Lane B, Torero JL. An Innovative Approach to Design Fires For Structural Analysis of NonConventional Buildings. In: Wald F, editor. Applications of Structural Fire Engineering. Prague, 2009.

[13] Rein G, Zhang X, Williams P, Hume B, Heise A, Jowsey A, Lane B, Torero JL. Multi-story Fire Analysis for High-Rise Buildings. Proceedings of the 11th International Interflam Conference; http://hdl.handle.net/1842/1980 (accessed on July 2010). London, 2007.

[14] Stern-Gottfried J, Rein G, Torero JL. Travel Guide. Fire Risk Management, 2009. p.12.

[15] Stern-Gottfried J, Rein G, Bisby LA, Torero JL. Experimental review of the homogeneous temperature assumption in post-flashover compartment fires. Fire Safety Journal 2010;45:249.

[16] Jonsdottir A, Rein G. Out of Range. Fire Risk Management, 2009. p.14.

[17] Fletcher I, Welch S, Capote JA, Alvear D, Lazaro m. Model-Based Analysis of a Concrete Building Subjected to Fire.

Advanced Research Workshop on Fire Computer Modelling. Santander, Spain, 2007.

[18] Gann RG. Reconstruction of the Fires in the World Trade Center Towers. NIST NCSTAR 1-5, 2005.

[19] NIST. Structural Fire Response and Probable Collapse Sequence of World Trade Centre Building 7 Volume 1. National Institute of Standards and Technology, 2008.

[20] Zannoni M. Brand bij Bouwkunde. COT Instituut voor Veilingheids - en Crisismanagement, 2008.

[21] Abecassis-Empis C, Reszka P, Steinhaus T, Cowlard A, Biteau H, Welch S, Rein G, Torero JL. Characterisation of Dalmarnock fire Test One. Experimental Thermal and Fluid Science 2008;32:1334.

[22] Welch S, Jowsey A, Deeny S, Morgan R, Torero JL. BRE large compartment fire tests--Characterising post-flashover fires for model validation. Fire Safety Journal 2007;42:548.

[23] Stern-Gottfried J, Law A, Rein G, Gillie M, Torero JL. A Performance Based Methodology Using Travelling Fires for Structural Analysis. 8th International Conference on Performance-Based Codes and Fire Safety Design Methods. Lund, 2010.

[24] Law A, Stern-Gottfried J, Gillie M, Rein G. The Influence of Travelling Fires on the Response of a Concrete Frame. International Conference of Structures in Fire. Lansing, Michigan, 2010.

[25] Audoin L, Kolb G, Torero JL, Most JM. Average Centerline Temperatures of a Buoyant Pool Fire Obtained by Image Processing of Video Recordings. Fire Safety Journal 1995;24:107.

[26] Alpert RL. Calculation of Response Time of Ceiling-Mounted Fire Detectors. Fire Technology 1972;8:181.

[27] Kodur VKR, Harmathy TZ. Properties of Building Materials. SFPE Handbook of Fire Protection Engineering. Quincy, MA, 2008.

[28] CEB-FIB. Fire Design of Concrete Structures: Structural Behaviour and Assessment. Lausanne: FiB, 2008.

[29] EN1992-1-1. Eurocode 2: Design of Concrete Structures - Part 1-1: General rules and rules for buildings, 1999.

[30] EN1992-1-2. Design of Concrete Structures - Part1-2: General rules- Structural fire design, 1992.

[31] EN1991-1-2. Eurocode 1: Actions of Structures - Part 1-2: General Actions - Actions on Structures Exposed to Fire,

1999.

[32] Abaqus. Abaqus Analysis User's Manual. Providence: Dassault Systemes Simulia Corp, 2008.

[33] Lubliner J, Oliver J, Oller S, Onate E. A Plastic-Damage Model for Concrete. International Journal of Solids and Structures 1989;25:299.

[34] Jonsdottir AM, Stern-Gottfried J, Rein G. Comparison of Resultant Steel Temperatures using Travelling Fires and Traditional Methods: Case Study for the Informatics Forum Building. 12th International Interflam Conference. Nottingham, 2010.

[35] Roben C. The effect of cooling and non-uniform fires on structural behaviour. School of Engineering, vol. PhD.

Edinburgh: The University of Edinburgh, 2009. 